\title{
Magnetic activity of six young solar analogues II. Surface Differential Rotation from long-term photometry
}

\author{
S. Messina ${ }^{1, \star}$ and E. F. Guinan ${ }^{2}$ \\ 1 Catania Astrophysical Observatory, INAF, via S. Sofia 78, 95123 Catania, Italy \\ e-mail: sme@ct.astro.it \\ 2 Dept. of Astronomy and Astrophysics, Villanova University, Villanova 19085, PA, USA \\ e-mail: edward.guinan@villanova.edu
}

A\&A, 409, 1017-1030 (2003), DOI: 10.1051/0004-6361:20031161

Key words. stars: activity - stars: late-type - stars: magnetic fields - stars: rotation - stars: starspots - errata, addenda

By mistake, the upper right panel of Fig. 1 has been printed five times. The correct figure is given on the next page.

^ Guest User, Canadian Astronomy Data Center, which is operated by the Dominion Astrophysical Observatory for the National Research Council of Canada's Herzberg Institute of Astrophysics. 

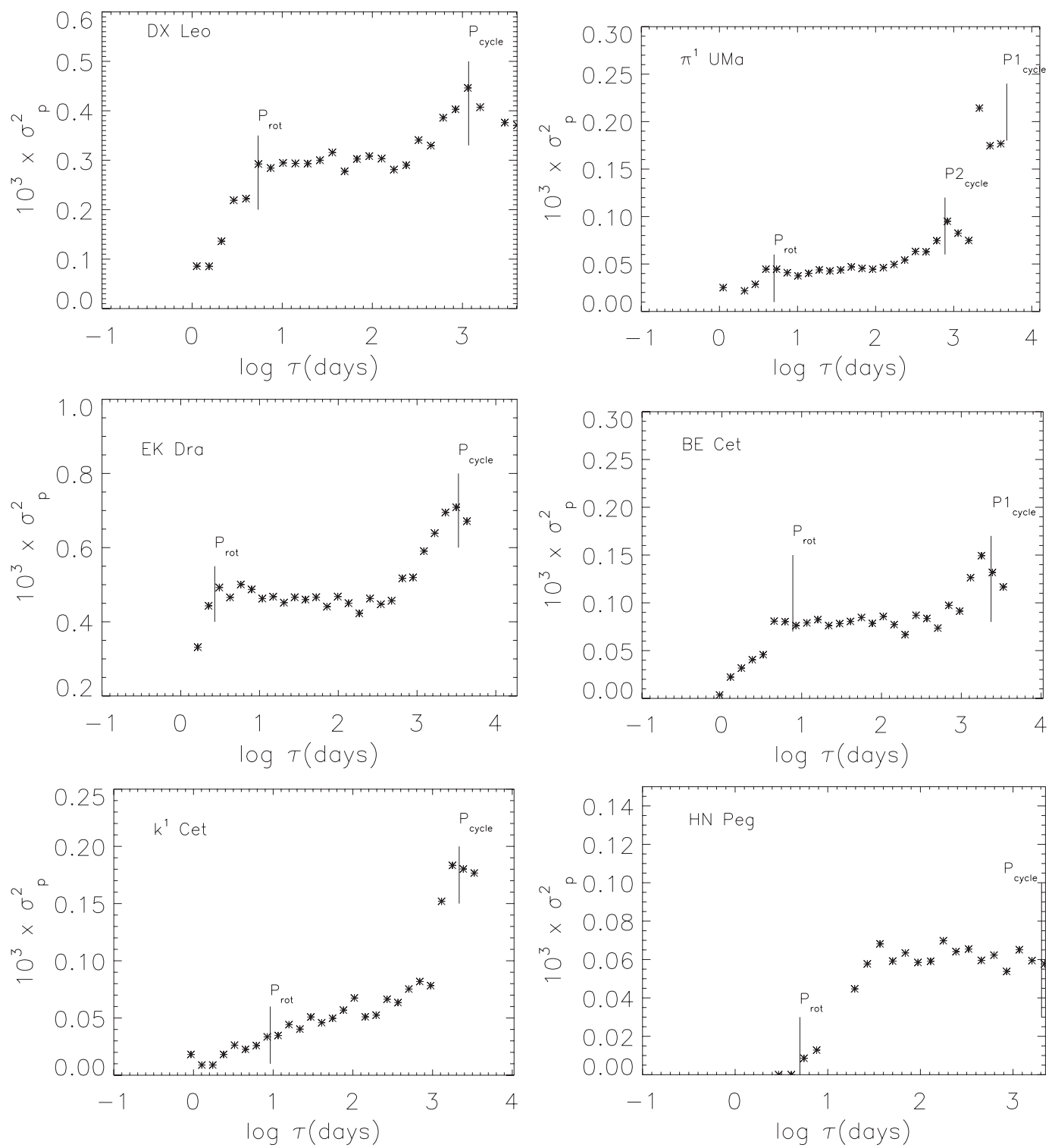

Fig. 1. Pooled variance profiles for program stars. The time scales of the rotation period modulation and of activity cycles are indicated by vertical bars. DX Leo, $\pi^{1}$ UMa, EK Dra and BE Cet are stars with distinct plateau according to Donahue et al. (1997a, Sol. Phys., 171, 211; 1997b, Sol. Phys., 171, 191), while HN Peg and $\mathrm{k}^{1}$ Cet are AR evolution-dominated stars. 